\title{
Issues of anamorphic fungi
}

\author{
Kevin D. Hyde
}

Published online: 12 October 2010

(C) Kevin D. Hyde 2010

\section{Fungal diversity -in this issue}

At the time when there is a move towards the acceptance of one name for one biological fungal species, Fungal Diversity documents the importance of anamorphic fungi with a special issue devoted to them. The present issue comprises 13 papers devoted to various topics concerning the anamorphic fungi with contributions on phylogeny, chemistry, ecology, post harvest importance, molecular detection and descriptions of some plant pathogens.

The first paper is a review of the biogeography and phylogeography of Fusarium. This important paper questions several trends in the understanding of this important genus which causes a wide variety of plant diseases, produces a number of mycotoxins and is becoming increasingly recognized as a significant human pathogen. The authors look at several examples where surveys of non agro-systems question the present understanding of this extraordinary genus and must be read. The second paper is also a review of the chemical and bioactive producing capabilities of the remarkable genus Pestalotiopsis. This mostly endophytic genus is especially productive with regard to the accumulation of a diverse array of mostly bioactive compounds. More than 130 different compounds

K. D. Hyde $(\bowtie)$

School of Science, Mae Fah Luang University,

Tasud,

Chiang Rai 57100, Thailand

e-mail: kdhyde2@gmail.com

K. D. Hyde

Botany and Microbiology Department, College of Science,

King Saud University,

Riyadh, Saudi Arabia with antifungal, antimicrobial, and antitumor activities have been isolated from various species of this amazing genus.

The third and fourth papers deal with post harvest topics. Colletotrichum gloeosporioides was previously reported to be the casual agent of anthracnose of most tropical fruits. This taxon, however, was recently epitypified and has been shown to be a species complex. A molecular study of isolates from Laos and Thailand causing anthracnose of eight tropical fruits shows that species other than $C$. gloeosporioides are responsible for anthracnose of most tropical fruits. This astounding result illustrates an urgent need to carry out research on re-inventory of tropical plant pathogens and should result in an unprecedented increase in phytopathogen research. Thirty one species belonging to 17 fungal genera were found to be associated with sorghum grain samples imported to the Kingdom of Saudi Arabia. These anamorphic fungi are important post harvest organisms producing important mycotoxins. The papers recommends that rigorous quarantine and healthy storage conditions should be undertaken to minimize fungal contamination and prevent further hazard to human and animal health.

Papers five to seven deal with assessing fungal biodiversity from environmental samples using molecular analysis. Sette et al. profiled the fungal community structure found in a Brazilian energy transmission tower with signs of corrosion and/or biofilm formation using cloning (ITS-rRNA gene libraries), a culture-dependent technique. A total of 31 isolates comprising ten filamentous fungi and four yeasts were recovered from enrichment cultures showing the usefulness of this method. Klaubauf et al. were also successfully able to use RFLP and sequence analysis of clone libraries of the partial ITS/LSU-region as a culture-independent method to survey fungal diversity in four arable soils and one grassland in Lower Austria. Seena et al. show that aquatic hyphomycetes can be directly 
identified using the ITS1-5.8S-ITS2 rRNA gene region or its subregions (ITS1 and ITS2) in their DNA barcoding of fungi: a case study using ITS sequences for identifying aquatic hyphomycete species.

The remaining six papers deal with various important groups of anamorphic fungi based on morphology, sequence analysis and other polyphasic approaches. Cheewangkoon et al resolve taxonomic position of Cryptosporiopsis eucalypti based on morphology and phylogenetic inference. C. eucalypti is shown to represent a new genus closely related to Plagiostoma for which the names Pseudoplagiostoma gen. nov. and Pseudoplagiostomaceae fam. nov. (Diaporthales) are introduced. Two new species of Cryptosporiopsis (Dermateaceae, Helotiales) on Eucalyptus from Australia and California (USA) are also described. Diogo et al. investigate Diaporthe and Phomopsis on almond in Portugal, which are important pathogens. They identified three species of which Phomop- sis amygdale is epitypified. Houbraken et al. investigate Penicillium citrinum and related species using a combination of partial $\beta$-tubulin, calmodulin and ITS sequence data, extrolite patterns and phenotypic characters. They conclude that seven species belong to this industrially important series and provide details. Kirschner and Chen report on three Periconiella species from Taiwan which includes one new species, while Walsh et al describe two new endophytic Fusarium species from tropical grasses of northern Australia. In the final paper Shenoy et al revisit the anamorphic genera Bahusutrabeeja, Diplococcium, Natarajania, Paliphora, Polyschema, Rattania and Spadicoides and elaborate their taxonomic placement. They recommend that that "where possible all new species descriptions, whether teleomorphic or anamorphic or pleomorphic, should include DNA sequence-data to facilitate amalgamation of anamorphic and pleomorphic genera in a single phylogenetic classification system". 